\title{
Suplementação de touros com sabões cálcicos de ácidos graxos poli-insaturados e qualidade seminal pré e pós-congelação
}

\section{Bulls supplemented with calcium soaps of polyunsaturated fatty acids and semen quality before and after freezing}

\author{
Mónica Marcela Ramírez HERNÁNDEZ ${ }^{1}$; Harold Ospina PATINO ${ }^{2 *}$; Ricardo Macedo GREGORY ${ }^{1}$; \\ Juan Camilo ANGEL ${ }^{2}$; Diogo del RE²; Maria Inês Mascarenhas JOBIM ${ }^{1}$; Rodrigo Costa MATTOS ${ }^{1}$ \\ ${ }^{1}$ Programa de Pós-graduação em Ciências Veterinárias da Faculdade de Medicina Veterinária da Universidade Federal de Rio \\ Grande do Sul, Porto Alegre-RS, Brasil \\ ${ }^{2}$ Programa de Pós-graduação em Zootecnia da Faculdade de Agronomia da Universidade Federal de Rio Grande do Sul, Porto \\ Alegre-RS, Brasil
}

\begin{abstract}
Resumo
O objetivo deste experimento foi avaliar a qualidade seminal de touros suplementados com sabões cálcicos de ácidos graxos poli-insaturados (PUFA). Vinte touros das raças Angus, Brangus, Hereford e Braford foram distribuídos aleatoriamente em dois grupos, cada um com dez animais. Cada grupo recebeu uma dieta composta de volumoso, ração comercial, sal mineral e um de dois suplementos: funcional com a incorporação de sabões cálcicos de PUFA ou energético, com a incorporação de raspa de mandioca (Manihot esculenta, Crantz). Durante o período experimental, a qualidade seminal foi avaliada utilizando testes rotineiros (volume, concentração, motilidade em massa, motilidade individual e avaliação da morfologia espermática) e testes complementares (teste hiposmótico, coloração dupla giemsa/tripan-azul e teste de termorresistência). O sêmen in natura do grupo de touros suplementados com sabões cálcicos de PUFA comercial (Megalac-E $)^{\circledast}$ apresentou aumento na motilidade espermática (83,3\% vs. 75,3\%), na percentagem de espermatozoides com acrossoma íntegro (94,8\% vs. $91,8 \%)$ e na percentagem de espermatozoides viáveis (98,0\% vs. 96,6\%) quando comparado com o sêmen do grupo de touros suplementados com raspa de mandioca $(\mathrm{P}<0,05)$. $\mathrm{O}$ tipo de suplemento não afetou o volume seminal, a concentração espermática e concentração de testosterona em sangue $(\mathrm{P}>0,05)$. Foram encontradas interações entre tratamento e número de coleta para percentagem de espermatozoides morfologicamente normais e percentagem de espermatozoides positivos ao teste hipo-osmótico $(\mathrm{P}<0,05)$. Após a descongelação, o tipo de suplemento não afetou a motilidade dos espermatozoides, o teste hipo-osmótico e o teste de termoresistência rápido $(\mathrm{P}>0,05)$. $\mathrm{A}$ suplementação com sabões cálcicos de PUFA aumentou as percentagens de espermatozoides com acrossoma íntegro (48,0\% vs. 39,2\%) e de espermatozoides com membrana plasmática integra (51,5\% v.s 42,2\%) pós-descongelação.
\end{abstract}

Palavras-chave: Touros. Sabões cálcicos. Ácidos graxos poli-insaturados. Características seminais. Raspa de mandioca.

\begin{abstract}
The objective of this experiment was to evaluate semen quality of bulls supplemented with calcium soaps of fatty acids polyunsaturated. Twenty Angus, Brangus, Hereford and Braford bulls were randomly allocated into two groups, each with ten animals. Each group received a diet consisting of forage, commercial ration, mineral supplements and supplement. Two supplements types were evaluated: functional supplement, with fatty acids polyunsaturated (PUFA) incorporation or energy supplement, with cassava meal (Manihot esculenta, Crantz) incorporation. During trial period the semen quality was evaluated using routine test (volume, concentration, mass motility, individual motility, sperm morphology) and complementary test: (hypo-osmotic test, giemsa/tripan-azul coloration and thermotolerance test). The semen of group bulls supplemented with commercial calcium soaps of PUFA (Megalac-E ${ }^{\circledast}$ ) showed greater sperm motility (83.3\% vs. $75.3 \%)$, percentage of sperm with intact acrosome (94.8\% vs. $91.8 \%)$ and percentage of sperm with intact plasma membrane $(98.0 \%$ vs. $96.6 \%)$ when compared with semen of group bulls supplemented with cassava $(\mathrm{P}<0.05)$. The supplement type did not affect semen volume, sperm concentration and testosterone concentration in blood $(\mathrm{P}>0.05)$. There were interactions between treatment and collection period for percentage of normal sperm and percentage of spermatozoa with hypo-osmotic positive test $(\mathrm{P}<0.05)$. The type of supplement did not affect sperm motility, the hypo-osmotic test and fast thermotolerance test after thawing $(\mathrm{P}>0.05)$. PUFA calcium soaps supplementation increased percentage of spermatozoa with plasma membrane integrity (51.5 vs. $42.2 \%)$ and spermatozoa intact acrosome (48.0\% vs. $39.2 \%)$ after thawing.
\end{abstract}

Keywords: Bulls. Calcium soaps. Polyunsatured fatty acids. Seminal characteristics. Cassava meal.

Correspondência para:

Harold Ospina Patino

Universidade Federal de Rio Grande do Sul (UFRGS)

Faculdade de Agronomia, Departamento de Zootecnia

Av. Bento Gonçalves, 7712, Agronomia
CEP: 91540-000, Porto Alegre, RS, Brasil

e-mail: harold.patino@ufrgs.br

Recebido: 08/07/10

Aprovado: 20/12/12 


\section{Introdução}

As biotécnicas da reprodução assistida são ferramentas atuais cada vez mais utilizadas nos sistemas de produção de bovinos na busca de eficiência reprodutiva. Deste modo, torna-se cada vez mais importante o entendimento dos fatores que podem influenciar os resultados em tais sistemas, entre eles a qualidade do sêmen ${ }^{1}$.

A qualidade do sêmen afeta diretamente o desempenho reprodutivo do touro ${ }^{2,3}$, pois as características seminais exercem um efeito importante desde o momento que os espermatozoides entram no trato reprodutivo da fêmea até o momento da fecundação ${ }^{4}$. Estudos têm detectado variações na fertilidade a partir de diferentes características seminais ${ }^{5}$ e mais recentemente alguns autores têm encontrado relações entre a concentração espermática, percentual de espermatozoides morfologicamente normais e a motilidade ${ }^{3,6}$.

O nível energético tem sido abordado nos estudos da influência da nutrição sobre a produção, reprodução e qualidade seminal, sendo mais importante nos animais em desenvolvimento destinados a serem futuros reprodutores ${ }^{7,8}$. Atualmente os estudos da influência da nutrição sobre a fertilidade bovina têm focado nas estratégias nutricionais para aumentar a qualidade dos espermatozoides nos machos e resposta nos programas de sincronização e qualidade do embrião nas fêmeas ${ }^{9}$. A suplementação com ácidos graxos tem sido orientada ao incremento do aporte energético na dieta, com o objetivo de melhorar o balanço energético. Recentemente, além da energia proporcionada pelos ácidos graxos, busca-se os benefícios que eles possuem como alimento funcional ${ }^{10}$.

Estudos demonstram que a suplementação de machos com ácidos graxos poli-insaturados melhora a qualidade seminal e incrementa os precursores de hormônios necessários para os processos reproduti$\operatorname{vos}^{11,12}$. Em touros, os ácidos graxos poli-insaturados (PUFA) podem melhorar a integridade e viabilidade da membrana espermática, além disso, Kelso et al. ${ }^{13}$ encontraram diminuição das concentrações de ácidos graxos poli-insaturados no plasma seminal com o envelhecimento dos indivíduos. Desta maneira, torna-se muito importante avaliar os possíveis benefícios da suplementação com PUFA sobre a qualidade seminal em touros.

O objetivo do presente estudo foi avaliar as características do sêmen de touros suplementados com sabões cálcicos de PUFA, determinando o seu efeito sobre a qualidade seminal pré e pós-descongelação.

\section{Material e Método}

\section{Local e duração do experimento}

O experimento foi realizado na central de processamento e congelamento de sêmen PROGEN', localizada no município de Dom Pedrito, RS, Brasil. O período experimental teve uma duração de 76 dias e foi iniciado com a pesagem, avaliação andrológica e início da suplementação dos animais. Foram realizadas cinco coletas de sêmen a cada quinze dias para avaliar a qualidade do sêmen in natura e, posteriormente, o sêmen foi congelado e avaliado pós-descongelação. As avaliações do sêmen in natura foram realizadas na PROGEN $^{\circ}$ enquanto as avaliações pós-descongelação foram realizadas no Laboratório de Reprodução Animal (REPROLAB) do Departamento de Medicina Animal da UFRGS.

\section{Tratamentos}

Avaliou-se o efeito de dois tipos de suplementos energéticos fornecidos para touros alimentados individualmente com volumosos cortados (pastagem de aveia ou cultivadas), ração comercial e sal mineral. Os tratamentos avaliados foram os seguintes:

Suplemento funcional: $200 \mathrm{~g} / \mathrm{d}$ de sabões cálcicos de ácidos graxos poli-insaturados (Megalac-Eº.

(Fornecida por QGN Química Geral do Nordeste, Brasil) e

Suplemento energético: $745 \mathrm{~g} / \mathrm{d}$ de raspa de Mandioca (Manihot esculenta, Crantz). 
As dietas foram isoenergéticas (Tabela 1); a composição dos ingredientes das dietas está apresentada na tabela 2; enquanto, a razão entre a quantidade administrada de cada ingrediente dietário e o peso vivo dos animais está na tabela 3 .

\section{Animais e Manejo}

Foram utilizados vinte touros das raças Angus, Brangus, Hereford e Braford, com peso médio de 950 kg e idade entre 4 e 10 anos. Cada animal foi considerado uma unidade experimental e alocado em um dos tratamentos mediante um processo de aleatorização estratificada, de modo a existirem animais de diferentes raças e idades similares dentro dos tratamentos.

Os touros estiveram alocados em vinte piquetes individuais de um hectare cada, divididos com cerca elétrica, cochos e bebedouros individuais. Os animais receberam volumoso verde cortado duas vezes ao dia e suplementação (fontes energéticas, ração comercial e sal) uma vez ao dia.

\section{Coletas de sêmen}

Foram realizadas cinco coletas de sêmen, sendo que a primeira foi feita ao início do experimento e tomada como referência pré-experimental. Posteriormente,

Tabela 1 - Consumo estimado da dieta dos animais durante o período experimental (Laboratório de Nutrição Animal, Departamento de Zootecnia, UFRGS)

\begin{tabular}{lcc}
\hline & Suplemento Funcional & Suplemento Energético \\
\hline Consumo de Matéria Seca (Kg) & 11,7 & 12 \\
Energia Metabolizável (Mcal/Kg) & 2,98 & 2,8 \\
Nutrientes Digestíveis Totais (\%) & 83 & 77,4 \\
Proteína bruta (\%) & 10,1 & 9,7 \\
\hline NRC Requerimentos Nutricionais gado de corte 1996 &
\end{tabular}

Tabela 2 - Composição bromatológica e componentes dos ingredientes utilizados na formulação das dietas (Laboratório de Nutrição Animal, Departamento de Zootecnia, UFRGS)

\begin{tabular}{|c|c|c|c|c|}
\hline & Pastagem & Ração & Megalac- $E^{\infty}$ & Mandioca \\
\hline MS \% & 36,5 & 80,9 & 95,6 & 87,3 \\
\hline PB \% & 8,0 & 17,6 & 0,4 & 3,3 \\
\hline $\mathrm{EE} \%$ & 3,1 & 4,3 & 44,2 & 0,5 \\
\hline FDA \% & 27,8 & 15,0 & - & 4,0 \\
\hline FDN \% & 54,0 & 15,1 & - & 11,1 \\
\hline LIG \% & 6,7 & 4,7 & - & 1,6 \\
\hline Ácido Linoléico \% & & & 41 & \\
\hline Ácido Linolênico \% & & & 2,8 & \\
\hline E.M (Mcal/kg) & & & 9,27 & 2,63 \\
\hline E.M Consumo (Mcal) & & & 1,77 & 1,71 \\
\hline
\end{tabular}

Tabela 3 - Componentes da dieta subministrada aos animais durante o período experimental em percentagem de peso vivo (\% PV). Central de Coleta e Processamento de sêmen, PROGEN

\begin{tabular}{lcc}
\hline & Suplemento Funcional & Suplemento Energético \\
\hline Megalac- $\mathrm{E}^{\oplus}(\% \mathrm{PV})$ & 0,02 & - \\
Raspa de Mandioca (\%PV) & - & 0,07 \\
Ração Comercial (\%PV) & 0,2 & 0,2 \\
Pastagem (\%PV) & 2,5 & 2,5 \\
Sal Mineral (\%PV) & 0,01 & 0,01 \\
\hline
\end{tabular}


os animais foram adaptados às dietas experimentais durante 15 dias. Após este período de adaptação, foram realizadas quatro coletas de sêmen com intervalo aproximado de 15 dias. O sêmen foi coletado em vagina artificial, após exposição do touro a uma vaca manequim. A qualidade seminal foi avaliada quanto: o volume do ejaculado, concentração espermática por meio de espectrofotometria, a motilidade e o vigor por meio de microscopia óptica comum e a percentagem de anormalidades utilizando microscopia de contraste de fase, utilizando as metodologias propostas pelo Colégio Brasileiro de Reprodução Animal ${ }^{14}$.

Para congelação as amostras de sêmen foram diluídas em citrato-gema com $4 \%$ de glicerol, envasadas em palhetas de $0,5 \mathrm{~mL}$ com uma concentração de $40 \times 10^{6}$ espermatozoides totais por dose, identificadas e colocadas em congelador automático MT $16812 / 0000^{\circ}$ em curva automática de congelação entre $-5^{\circ} \mathrm{C}$ a $-120^{\circ} \mathrm{C}$, duração do ciclo dez minutos e armazenadas em nitrogênio liquido a $-196^{\circ} \mathrm{C}$ para posteriores avaliações pós-descongelação. As partidas de sêmen foram descongeladas e avaliadas quanto a sua motilidade progressiva retilínea em microscópio óptico aumento 100X, integridade do acrossoma e diferenciação de espermatozoides viáveis de mortos por meio de uma coloração dupla (Tripan azul e Giemsa) que permite fazer uma avaliação simultânea de ambas as carateristicas ${ }^{2}$, de integridade da membrana plasmática por meio do teste hiposmótico $(\mathrm{HO})^{15,16}$, submetendo aos espermatozoides a uma solução de $100 \mathrm{Mosm} / \mathrm{L}$ de citrato de sódio e frutose e avaliando cauda enrolada de não enrolada em microscópio óptico em aumento de 1000X, e longevidade dos espermatozoides pelo teste de termorresistência rápido $(\mathrm{TTR} / \mathrm{R})^{17}$ avaliando motilidade progressiva retilínea por microscopia óptica no momento da descongelação, e depois de um período de incubação de 30 e 60 minutos em banho termostático a $45^{\circ} \mathrm{C}^{18}$.
Também foram tomadas e conservadas congeladas duas amostras de soro para determinação da concentração de testosterona sérica. As amostras de sangue para análise de concentração de testosterona foram coletadas por meio de Vacutainer e punção da veia coccígea em tubos sem anticoagulante para separação do soro. A concentração de testosterona em $\mathrm{ng} / \mathrm{mL}$ foi medida por meio de radioimunoensaio (Kit RIA CT) que mede o esteroide na sua forma não conjugada ${ }^{19}$.

Para determinação da qualidade das pastagens e suplementos fornecidos durante o período experimental, foram feitas análises de matéria seca, cinzas, proteína, gordura, fibra, fibra em detergente ácida (FDA), fibra em detergente neutro (FDN), lignina e energia. Dos suplementos foi coletada uma só amostra antes do início do experimento, das pastagens foram coletadas amostras para análise a cada vinte dias durante o período experimental.

\section{Analise Estatística}

$\mathrm{Na}$ análise dos dados utilizou-se a análise de variância com medidas repetidas no tempo para dois tratamentos e quatro coletas. A primeira coleta foi usada como co-variável nas análises estatísticas com o fim de igualar os grupos quanto à qualidade seminal. A estrutura da matriz de covariância que resulta em melhor ajuste do modelo foi escolhida com base nos menores valores para o Critério de Informação de Akaike (AIC) e Critério de Informação Bayesiano Swarz (BIC). As informações sobre as variáveis motilidade espermática, percentagem de acrossomas íntegros e motilidade dos espermatozoides submetidos ao TTR/R aos 30 e 60 minutos, foram transformadas em arco seno. O software utilizado foi o SAS e as medias comparadas pelo teste-t. $\mathrm{O}$ modelo matemático usado foi: Yijk $=\mu+\tau_{i}+t_{j}+\left(\tau^{\star} t\right)_{i j}+\varepsilon_{i j k}$; onde: $y_{i j k}=$ observação ijk-ésima observação; $\mu=$ média geral; $\tau_{i}=$ efeito do i-ésimo tratamento ( $\mathrm{i}=1$ e 2$) ; t_{j}=$ efeito da j-ésima coleta $(\mathrm{j}=1,2,3$ e 4$) ;\left(\tau^{\star} t\right)_{i j}=$ efeito da ij-ésima interação entre o tratamento e a coleta; $\varepsilon_{i j k}=$ efeito do ijk-ésimo associado a ijk-ésima observação. 


\section{Resultados}

O tipo de suplemento não afetou a concentração sérica de testosterona $(\mathrm{P}=0,688)$, sendo a média e desvio padrão de $4,11 \pm 2,15 \mathrm{mg} / \mathrm{mL}$ para touros suplementados com dieta funcional e de 4,73 $\pm 3,64 \mathrm{mg} /$ $\mathrm{mL}$ para aqueles suplementados com dieta energética.

Não foram notados efeitos do tipo de suplemento sobre o volume seminal $(\mathrm{P}=0,5963)$ nem sobre a concentração espermática $(\mathrm{P}=0,5117)$. Por outro lado, o sêmen dos touros alimentados com dietas contendo suplemento funcional teve incrementos de $11 \%$ na motilidade ( $\mathrm{P}=0,0443), 3 \%$ nos espermatozoides com acrossoma íntegro e 1,5\% nos espermatozoides com membrana plasmática íntegra $(\mathrm{P} \leq 0,05)$, quando comparado com o sêmen de touros recebendo suplemento energético (Tabela 4).

Foram encontradas interações entre tratamento e sessão de coleta para percentagens de espermatozoides com morfologia normal $(\mathrm{P}=0,0344)$ e $\mathrm{HO}$ positivos $(\mathrm{P}$ $=0,0168)$ mostradas, respectivamente, nas figuras 1 e 2 .

A motilidade espermática pós-descongelação não apresentou diferença entre os grupos suplementados. Os valores de espermatozoides com acrossoma íntegro foram superiores no grupo suplementado com sabões cálcicos de PUFA ( $\mathrm{P}=0,0034)$, o que também aconteceu para percentagem de espermatozoides com membrana plasmática intacta $(\mathrm{P}=0,0056)$, quando comparado com o grupo que recebeu suplemento energético (Tabela 4).

O tipo de suplemento energético não afetou $(\mathrm{P}>0,05)$ a longevidade espermática aos 30 ou aos 60 minutos de incubação, nem o teste hipo-osmótico pós-descongelação (Tabela 4).

\section{Discussão}

O tipo de suplemento energético utilizado neste estudo não afetou o volume, a concentração de espermatozoides nem a concentração sérica de testosterona, confirmando os resultados de outros trabalhos onde os animais foram suplementados com fontes de ácidos graxos poli-insaturados PUFA $^{12,20}$. Por outro lado, o sêmen in natura de touros alimentados com dietas contendo sabões cálcicos de PUFA teve incremento na motilidade espermática ( $83,3 \%$ vs. $75,3 \%)$, em concordância com vários estudos em diferentes espécies $^{21,22,23,24}$, onde a suplementação com fontes de Omega-3 apresentou correlação positiva com o número de espermatozoides móveis ${ }^{25}$, além disto, no

Tabela 4 - Média \pm desvios padrão das características seminais in natura e pós-criopreservação de touros alimentados com dietas contento suplementos funcional e energético

\begin{tabular}{|c|c|c|c|}
\hline Pârametros (\%) & $\begin{array}{l}\text { Suplemento } \\
\text { Funcional }\end{array}$ & $\begin{array}{l}\text { Suplemento } \\
\text { Energético }\end{array}$ & $p$ \\
\hline \multicolumn{4}{|l|}{ In natura } \\
\hline Volume (mL) & $6,81 \pm 0,33^{\mathrm{a}}$ & $6,42 \pm 0,43^{\mathrm{a}}$ & $\mathrm{P}=0,5963$ \\
\hline Motilidade espermática (\%) ${ }^{1}$ & $83,37 \pm 1,16^{\mathrm{a}}$ & $75.37 \pm 2,33^{b}$ & $P=0,0443$ \\
\hline Concentração espermática $\left(\mathrm{x} 10^{6} / \mathrm{mL}\right)$ & $997,53(56,8)$ & $1,064,53(46,1)$ & $P=0,5117$ \\
\hline Acrossomas íntegros (\%) ${ }^{1}$ & $94,86 \pm 0,35^{\mathrm{a}}$ & $91,86 \pm 0,56^{b}$ & $\mathrm{P}=0,0001$ \\
\hline $\begin{array}{l}\text { Espermatozoides com membrana } \\
\text { plasmática intacta (\%) }\end{array}$ & $98,05 \pm 0,25^{a}$ & $96,63 \pm 0,32^{b}$ & $\mathrm{P}=0,0013$ \\
\hline \multicolumn{4}{|l|}{ Pós-congelação } \\
\hline Motilidade espermática (\%) & $37,5 \pm 1,70^{\text {a }}$ & $30,5 \pm 1,80^{\text {a }}$ & $\mathrm{P}=0,1027$ \\
\hline Acrossomas íntegros (\%) & $48,0 \pm 1,80^{a}$ & $39,2 \pm 1,80^{b}$ & $\mathrm{P}=0,0034$ \\
\hline $\begin{array}{l}\text { Espermatozoides com membrana } \\
\text { plasmática intacta (\%) }\end{array}$ & $51,5 \pm 2,00^{a}$ & $42,2 \pm 2,00^{b}$ & $\mathrm{P}=0,0056$ \\
\hline
\end{tabular}




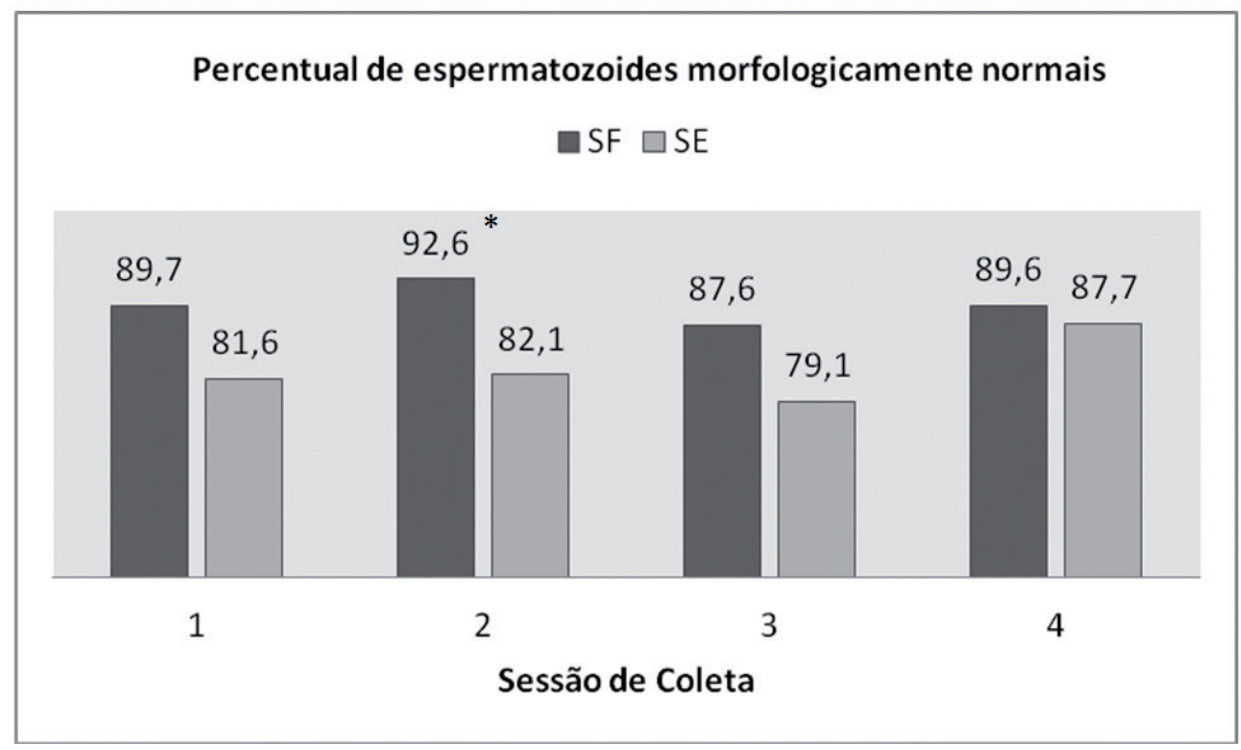

Figura 1 - Relação entre período da coleta e a percentual de espermatozoides morfologicamente normais em sêmen in natura de touros recebendo dois tipos de suplemento energético (SF: Suplemento Funcional; SE: Suplemento Energético). * Símbolo em cima das barras indica diferença significativa pelo teste-t $(\mathrm{P} \leq 0.001)$

A primeira coleta durante o período experimental foi tomada como co-variável quando significativa

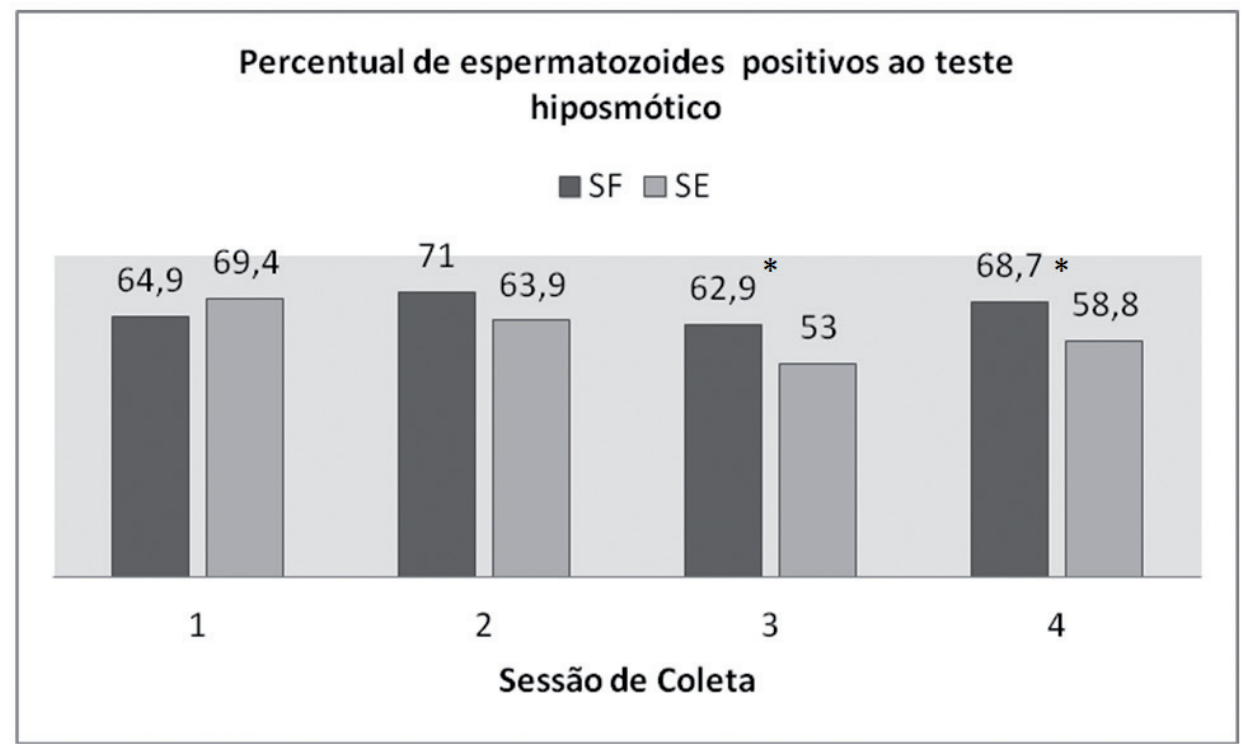

Figura 2 - Relação entre período da coleta e a percentual de espermatozoides que reagiram positivamente ao teste hipo-osmótico (HO) em sêmen in natura de touros recebendo dois tipos de suplemento energético (SF: Suplemento Funcional; SE: Suplemento Energético). * Símbolo em cima das barras indica diferença significativa pelo teste-t $(\mathrm{P} \leq 0.001)$

A primeira coleta durante o período experimental foi tomada como co-variável quando significativa

presente trabalho, houve incremento da percentagem de acrossomas íntegros (94,8\% vs $91,8 \%)$ e percentagem de espermatozoides viáveis (98,0\% vs $96,6 \%)$ em relação ao sêmen de touros recebendo suplemento energético. A análise multifatorial dos atributos da qualidade dos espermatozoides é a avaliação mais re- 
comendável e apropriada para o diagnóstico da funcionalidade e integridade estrutural das células, visando estimar a habilidade fecundante do sêmen ${ }^{26,27}$, mesmo que seja de maior importância no sêmen após descongelação ${ }^{18}$, um sêmen in natura em boas condições, quando a metodologia de diluição e congelação otimamente aplicada, é um sêmen de melhor qualidade pós-descongelação.

As avaliações feitas em amostras do sêmen após congelação mostrou incrementos superiores a $20 \%$ em percentagens de espermatozoides com membrana acrossomal íntegra (48,0\% vs 39,2\%) e percentagens de espermatozoides viáveis (51,5\% vs $42,2 \%)$. A suplementação de touros com sabões cálcicos de PUFA pode ajudar a diminuir as alterações morfológicas nas membranas dos espermatozoides induzidas pela criopreservação. Pettitt e Buhr ${ }^{28}$ reportaram modificações dos fosfolipídios do espermatozoide durante a criopreservação e posterior aquecimento, com maior dano sofrido pela membrana plasmática da cabeça. Posteriormente, Cerolini et al. ${ }^{29}$ acharam um decréscimo e uma mudança no arranjo dos fosfolipídios de espermatozoides submetidos a processos de congelação e descongelação.

A suplementação com ácidos graxos poli-insaturados tem mostrado efeitos positivos sobre a capacidade reprodutiva de machos adultos. A manipulação da dieta contendo PUFA muda a composição dos componentes dos espermatozoides de várias espécies domésticas ${ }^{30,31}$, melhorando as características físicas e metabólicas das membranas espermáticas, plasmática e acrossomal ${ }^{4}$. Dessa forma, pode se conferir homogeneidade aos espermatozoides e incrementar a possibilidade de que maior número chegue à junção útero-tubárica aumentando a probabilidade de fecundação $0^{4,32}$. Alguns estudos têm sugerido que a redução na motilidade e número de espermatozoides do ejaculado de touros podem ser decorrentes de deficiências de ácido docosaexaenóico (DHA) nos fosfolipídios da membrana espermática ${ }^{13}$. Sob as condições do presente estudo, pode se afirmar que a suplementação com sabões cálcicos de fontes de PUFA pode conferir às membranas espermáticas arranjos mais resistentes às injurias dos processos de criopreservação e posterior aquecimento.

Os touros do presente estudo pertencem a uma central de coleta e processamento de sêmen, submetidos segundo a demanda, a várias coletas no percurso da semana. Estudos avaliando os efeitos de alta frequência de coletas sobre a qualidade seminal encontraram efeitos sobre motilidade espermática, localização de gota citoplasmática e incremento de algumas anormalidades no epidídimo e ejacula$\mathrm{do}^{33,34}$. A percentagem de espermatozoides morfologicamente normais do sêmen in natura no presente experimento exibiu uma interação significativa com a segunda sessão de coleta durante o período experimental, sendo maior nos touros suplementados com fontes de ácidos graxos poli-insaturados. Demonstrando uma possível relação entre percentagem de espermatozoides normais com a frequência de coletas às que os touros estavam sendo submetidos além do período experimental (Figura 1). Devido à impossibilidade de controlar manejos no interior das centrais, recomendamos realizar futuros estudos usando espectrofotometria de massas MALDI ou MALDI-MS (Matrix-assisted laser desorption/ionization mass spectrometry), com o fim de estimar-se a efetiva incorporação dos ácidos graxos poli-insaturados nas membranas espermáticas.

Houve uma interação entre a percentagem de espermatozoides que reagiram positivamente ao teste hipo-osmótico no sêmen in natura e as últimas duas sessões de coleta. A resposta foi melhor nos espermatozoides de touros suplementados com fontes de ácidos graxos poli-insaturados (Figura 2). Estudos avaliando a correlação do teste hiposmótico positivo com outros parâmetros de qualidade como motilidade e percentagens de espermatozoides com acrossoma íntegro observaram correlação positiva entre estes parâme- 
tros, deduzindo que os testes de avaliação em conjunto demonstram a capacidade funcional dos espermatozoides no sêmen pré e pós-descogelamento ${ }^{15}$. No presente estudo, a percentagem de espermatozoides que reagiram positivamente ao teste hiposmótico no sêmen pós-descongelação não apresentou diferenças entre os grupos, porém, apresentou maiores percentagens em espermatozoides com membrana acrossomal íntegra e espermatozoides com membrana plasmática intacta, resultando em espermatozoides com maior capacidade funcional tanto no sêmen fresco como no sêmen pós-descogelação.

Segundo os resultados do presente estudo, a suplementação de touros com fontes de ácidos graxos poli-insaturados melhora a motilidade dos espermatozoi-

\section{Referências}

1. BAILEY, J. L.; BILODEAU, J. F.; CORMIER, N. Semen Cryopreservation in domestic animals: a damaging and capacitating phenomenon. Journal Andrology, Université Laval, Quebéc, Canada, v. 21, n. 1, p. 1-7, 2000.

2.BRITO, L. F.; BARTHE, A. D.; BILODEAUR-GOESEELS, S.; PANICH, P. L.; KASTELIC, J. P. Comparison of methods to evaluate the plasmalemma of bovine sperm and their relationship whit in vitro fertilization rate. Theriogenology, $v$. 60, n. 8, p. 1539-1551, 2003.

3. MOSTARI, M. P.; RAHMAN, M. G. M.; KHANDOKER, M. A. M. Y.; HUSAIN, S. S. Evaluation of bulls based on semen quality and herd fertility. Pakistan Journal of Biology Sciences, Bangladesh, v. 7, n. 12, p. 2177-2181, 2004.

4. PALMA, G. A.; MICELI, D. Interacción Funcional entre los espermatozoides y el tracto reproductor de la hembra. In: PALMA, G. A. Biotecnologia de la reproducción. 2. ed. Mar del Plata: Pugliese y Siena, 2008. p. 93-134.

5. NISHIKAWA, Y. Studies on reproduction in horses: singularity and artificial control in reproductive phenomena. Tokyo: Japan Racing Association, 1959. 340 p.

6. SARDER, M. J. U. Morphological sperm abnormalities of different breeds of AI bull and its impact on conception rate of cows in AI programme. Blanglandesh Journal of Veterinary Medicine, v. 2, n. 2, p. 129-135, 2004.

7. BARTH, A. D.; BRITO, L. F.; KASTELIC, J. P. The effect of nutrition on sexual development of bulls. Theriogenology, $v$. 70, n. 3, p. 485-494, 2008

8. FOOTE, R. H. Factors influencing the quantity and quality of semen harvested from bulls, rams, boars factors influencing the quantity and quality and stallions. Journal Animal Science, v. 47, p. 1-11, 1978. Supplement, 2.

9. ABAYASEKARA, D. R. E.; WHATES, D. C. Effects of altering dietary fatty acid composition on prostaglandin synthesis and fertility. Prostaglandins, Leukotrienes and Essential Fatty Acids, v. 61, n. 5, p. 275-288, 1999. des e as características das membranas espermáticas, aumentando o número de espermatozoides viáveis e com membrana acrossomal íntegra, sobretudo no sêmen após ser exposto a processos de criopreservação, aumentando assim o valor agregado às biotécnicas como a inseminação artificial, transferência de embriões e fertilização in vitro de embriões, onde geralmente é usado sêmen após criopreservação.

\section{Agradecimentos}

Agradecimentos à empresa Química Geral do Nordeste (QGN) pelo fornecimento de recursos financeiros e matérias primas para realização do experimento e à CAPES pela disponibilização da bolsa PEC - PG.

10.COLLA, L. M.; MORAES, F. P. Alimentos funcionais e nutracêuticos: definições, legislação e benefícios à saúde. Revista Electrônica de Famácia, v. 3, n. 2, p. 109-122, 2006.

11.McVEY, M. J.; COOKE, G. M.; CURRAN, I. H.; CHAN, H. M.; KUBOW, S.; LOK, E.; MEHTA, R. Effects of dietary fats and proteins on rat testicular steroidogenic enzymes and serum testosterone levels. Food and Chemical Toxicology, v. 46, n. 1, p. 259-269, 2007.

12.ARLAS, T. R.; PEDERZOLLI, C. D.; TERRACIANO, P. B.; TREIN, C. R.; BUSTAMANTE-FILHO, I. C.; CASTRO, F. S.; MATTOS, R. C. Sperm quality is improved feeding stallions with a rice oil supplement. Animal Reproduction Science, v. 107, n. 3, p. 306, 2008. Abstracts.

13.KELSO, K. A.; CEROLINI, S.; SPEAKE, B. K.; CAVALCHINI, L. G.; NOBLE, R. C. Effects of dietary supplementation with alpha-linolenic acid on the phospholipid fatty acid composition and quality of spermatozoa in cockerel from 24 to 72 weeks of age. Journal of Reproduction and Fertility, v. 110, n. 1, p. 53-59, 1997.

14. COLEGIO BRASILEIRO DE REPRODUÇÃO ANIMAL (CBRA). Manual para exame andrológico e avaliação de animal. 2. ed. Belo Horizonte: Colegio Brasileiro de Reprodução Animal, 1998. 53 p.

15.LODHI, L. A.; ZUBAIR, M.; QURESHI, Z. I.; AHMAD, I.; JAMIL, H. Correlation between hypo-osmotic swelling test and various conventional semen evaluation parameters in fresh nili-ravi buffalo and sahiwal cow bull semen. Pakistan Veterinary Journal, v. 28, n. 4, p. 186-188, 2008.

16. RODRÍGUEZ-GILA, J. E.; MONTSERRATA, A.; RIGAU, T. Effects of hypoosmotic incubation on acrosome and tail structure on canine spermatozoa. Theriogenology, v. 42 , n. 5, p. 815-829, 1994.

17.ARRUDA, R. P.; BARNABE, V. H.; ALENCAR, M. M. de; BARNABE, R. C. Avaliação de sêmen congelado de bovinos. Provas lenta e rapida de termo-resistência: efeitos sobre a 
fertilidade. Brazilian Journal Veterinary Research Animal Science, São Paulo, v. 29, n. 1, p. 131-137, 1998.

18.ARRUDA, R. L.; ORRO, I. R.; PASSOS, T. S.; COSTA E SILVA, E. V.; ZÚCCARI, C. E. S. N. Técnicas para avaliação laboratorial da integridade estrutural e funcional do sêmen congelado de touros. Revista Brasileira de Reprodução Animal, Belo Horizonte, v. 34, n. 3, p. 168-184, 2010.

19.CHEN, A.; BOOKSTEIN, J. J. Meldrum: diagnosis of a testosterone-secreting adrenal adenoma by selective venous catheterization. Fetility and Esterility, v. 55 , n. 6, p. $1202-$ $1203,1991$.

20.ADEEL, M.; IJAZ, A.; ALEEM, M.; REHMAN, H.; YOUSAF, M. S.; JABBAR, M. A. Improved of liquid and frozed-thawed semen quality of Nili-Ravi buffalo bulls (bubalus bubalis) throuhg supplementation of fat. Theriogenology, v. 71, n. 8, p. 1220-1225, 2009.

21.CONQUER, J. A.; MARTIN, J. B.; TUMMON, I.; WATSON, L.; TEKPETEY, F. Effect of DHA supplementation on DHA status and sperm motility in asthenozoospermic males. Lipids, v. 35, n. 2, p. 149-154, 2000.

22.DOLATPANAH, M. B.; TOWHIDI, A.; FARSHAD, A. RASHIDI, A.; REZAYAZDI, A. Effect of dietary fish oil on semen quality of goats. Asian-Australasian Journal Animal Sciences, v. 21, n. 1, p. 29-34, 2008.

23. GHOLAMI, H.; CHAMANI, M.; TOWHIDI, A.; FAZELI, M. $\mathrm{H}$. Improvement of semen quality in Holstein bulls during heat stress by supplementing of omega-3 fatty acids. World Academy of Science, Engineering \& Technology, v. 68, p. 939,2010

24. ROOKE, J. A.; SHAO, C. C.; SPEAKE, B. K. Effects of feeding tuna oil on the lipid composition of pig spermatozoa and in vitro characteristics of semen. Reproduction, v. 121, n. 2, p. 315-322, 2001.

25.TOWHIDI, A.; SAMADIAN, F.; REZAYAZDI, K.; ROSTAMI, F; GHAZIANI, F. Feeding n-3 fatty acid source improves semen quality by increasing $\mathrm{n} 3 / \mathrm{n} 6$ fatty acids ratio in sheep. In: WORLD CONFERENCE ON ANIMAL PRODUCTION, 10., 2008, Cape Town. Abstract... Netherlands: Wageningen Academic Publishers, 2008. p. 65.
26.LARSSON, B.; RODRÍGUEZ-MARTÍNEZ, H. Can we use in vitro fertilization tests to predict semen fertility? Animal Reproduction Science, v. 60/61, p. 327-336, 2000.

27. PARKINSON, T. J. Evaluation of fertility and infertility in natural service bulls. Veterinary Journal, v. 168, n. 3, p. 215229,2004

28.PETTITT, M. J.; BUHR, M. M. Extender components and surfactants affect boar sperm function and membrane behavior during cryopreservation. Journal of Andrology, v. 19, n. 6, p. 736-746, 1998.

29. CEROLINI, S.; MALDJIAN, A.; PIZZI, F.; GLIOZZI, T. M. Changes in sperm quality and lipid composition during cryopreservation of boar semen. Reproduction, Chambridge, v. 121, n. 3, p. 395-401, 2001.

30.BLESBOIS, E.; DOUARD, V.; GERMAIN, M.; BONIFACE, P.; PELLET, F. Effects of n-3 polyunsaturated dietary supplementation on the reproductive capacity of male turkeys. Theriogenology, v. 61, n. 2-3, p. 537-549, 2004.

31.PENNY, P. C.; NOBLE, R. C.; MALDJIAN, A.; CEROLINI, S. Potential role of lipids for the enhancement of boar fertility and fecundity. Ping News and Information, v. 21, n. 4, p. $119-126,2000$

32.SAACKE, R. G. Relevancia de características del semen compensables y no compensables en la fertilidad y la calidad embrionaria en los bovinos. In: SIMPOSIO INTERNACIONAL DE REPRODUCIÓN ANIMAL IRAC, 8., 2009, Cordoba, Buenos Aires.

33.PRUNEDA, A; PINART, E.; DOLOZ, B. M.; SANCHO, S.; GARCIA-GIL, N.; BADIA, E.; KÁDÁR, E.; BASSOLS, J.; BUSSALLEU, E.; YESTE, M.; BONET, S. Effects of a high semen-collection frequency on the quality of sperm from ejaculates and from six epididymal regions in boars. Theriogenology, France, v. 63, n. 8, p. 2219-2232, 2005.

34. BALL, P. J. H.; PETERS, A. R. Bull fertility. In: BALL, P. J. H.; PETERS, A. R. Reproduction in cattle. 3. ed. Oxford, UK: Blackwell Publishing, 2004. chap. 3, p. 28-39, 2004. 